\title{
A Real-time Tracking and Warning System for Civil Aircraft Flight Based on Fusion of the Multi-source data Information approach
}

\author{
Haibo $\mathrm{Ma}^{1, a}$, Yiping $\mathrm{YaO}^{1, \mathrm{~b}}$ \\ ${ }^{1}$ College of Information System and Management, National University of Defense Technology, \\ Changsha, 410073, China \\ aemail: mahaibo168@126.com, bemail: ypyao@nudt.edu.cn
}

Keywords: Flight Real-time Tracking; Civil Aircraft Safety; Multi-source data fusion; MH370

\begin{abstract}
The crash occurred frequently recent years, civil aviation aircraft safety issues become the focus of attention, and real-time tracking and monitoring of flight trajectory has become an urgent problem to be solved. At present, Aircraft Communication Addressing and Reporting System (ACARS) is the only accurate and reliable technology for the aircraft to obtain the position. However, the technology has the imperfections such as poor timeliness, passive monitoring and so on. In addition, there is no research on the method of real-time intelligent judgment and early warning for abnormal behaviors through flight trajectory. To address these problems, this paper modeled based on multi-source data information of ACARS, ADS-B (Automatic Dependent Surveillance-Broadcast), planed route, history of flight and meteorological data, and then firstly proposed the real time tracking and monitoring approach based on fusion of multi-source data information for civil aircraft, which consists of three modules: (1) visual real-time tracking and monitoring (2) route deviation alarm (3) position prediction when lose contact in short time. Based on this approach, the flight tracking and warning system of civil aircraft is implemented. The practical application proves that the approach and the model of the technology is feasible.
\end{abstract}

\section{Introduction}

In recent two years, several airliners lost contact or crash, triggered a global hot spots for civil aviation aircraft flight safety concerns, but also exposed the existing business aviation industry technology is actually not like the outside world think that airlines on every aircraft flight have effective strategies for real time management and monitoring. There is an urgent issue to be solved $\mathrm{f}$ to monitor and manage thousands of flights per day in real-time as well as automatically.

How can we prevent mishaps, errors, and chain of events before they cause a major disaster? How can we prevent the dangers of human errors in panic similar to Air France 447 and possibly for Malaysian Airline MH370, rather than analyze what happened after a crash, and only if the black box was found?

Requirements are summed up on three conditions for the aircraft flight tracking and monitoring: (1) the aircraft mechanical failure (2) an irresistible disaster like weather, etc. (3) abnormal flight behavior caused by individual pilot. First two situation is often solve through the technical means tend to safe and controllable, but for the third point there has not efficient way. The recent several situation of civil aircraft losing contact, exactly occurred due to unable to monitor aircraft flight behavior, thus missed the chance to locate the aircraft at the most precious period and caused huge cost and difficulty for later investigation and searching work[1].

In normal circumstances, the flight crew will strictly comply with the airline's scheduled flight route. However in practice, the flight crew will often decide which deviated from the route, and then return to the predetermined route due to weather the area, save fuel or to avoid delays and other considerations[2]. This is indeed the need for existence. But this is also the biggest risk in the flight behavior of the aircraft. Because the existing monitoring mechanism determines, that the flight crew completely control the flying status by individually after the former ground permission to take off while before the next air traffic control unit to take over it. If there happens accident off course, the situation is that only the crew themselves know while the ground commander get 
nothing. Ground personnel can only be received by the pilot to report anomaly. But if the crew does not report, it is unable to find abnormal for the ground. This is why MH370 is not noticed in the yaw nearly 40 minutes before found abnormal, thus lost contact with the ground. Each airline has a large number of flights, therefore, flight monitoring and security issues are particularly important and there are urgent demand for tracking and early warning the flight status of each aircraft.

To avoid missing monitoring abnormal behavior of the aircraft flight in the lag time, there should be second frequency real-time monitoring, judgment and alarm for the flight behavior anomaly.

Most of the existing tracking technologies rely mainly on ACARS data[3] to report the aircraft position. However, ACARS often interval of half an hour or longer, so it is low frequency, Lacking timeliness and followed by passive monitoring. Because control personnel receives data passively by recording devices to send ACARS data. ADS-B (automatic dependent Surveillance-Broadcast) [4] is also tracking technology that has high frequency but low accuracy. Unfortunately, neither of these two technologies support real-time intelligent early warning yawing abnormal flight behavior. Some advanced military radar can indeed achieve real-time monitoring of any aircraft, but military radar technology and data is unavailable or realistic to share with the civil aviation company.

Motivated to solve the above problems, this paper modeled for fusion of the ACARS data, ADS-B data, planed route data, flight history data and meteorological data ,and then proposed a real time tracking and warning approach based on fusion of the Multi-Source data information, which contains three modules: (1) visual real-time monitoring (2) off course deviation alarm (3) briefly lost position prediction. Based on the approach, we implemented the civil aircraft flight tracking alarm system. The system data interface is provided by cooperation of China Southern Airlines, which has been already applied to the actual business of the company to monitor all flights.

Section 2 introduces the existing aircraft flight tracking related technologies. Section 3 presents details of the aircraft real-time tracking alarm approach based on the fusion of multi-source information. Section 4 introduces implement of real-time tracking and warning system for civil aircraft flight. Finally, our conclusion will be made with a summary of the research work.

\section{Existing aircraft flight tracking related technologies}

\section{ACARS technology.}

With the development of civil aviation, the airlines need more and more data to support their operation and maintenance requirements, and the air traffic management departments need more data to improve their communication and surveillance capabilities. The airlines, in an effort to reduce crew workload and improve data integrity, introduced the Aircraft Communication Addressing and Reporting System (ACARS) in the late 1980s. And all civil aircraft uses ACARS technology as the major mains of communication with the ground. ACARS is a data-link system which allows communication of character-oriented data between aircraft systems and ground systems via very high frequency (VHF) communication or satellite communication [5].

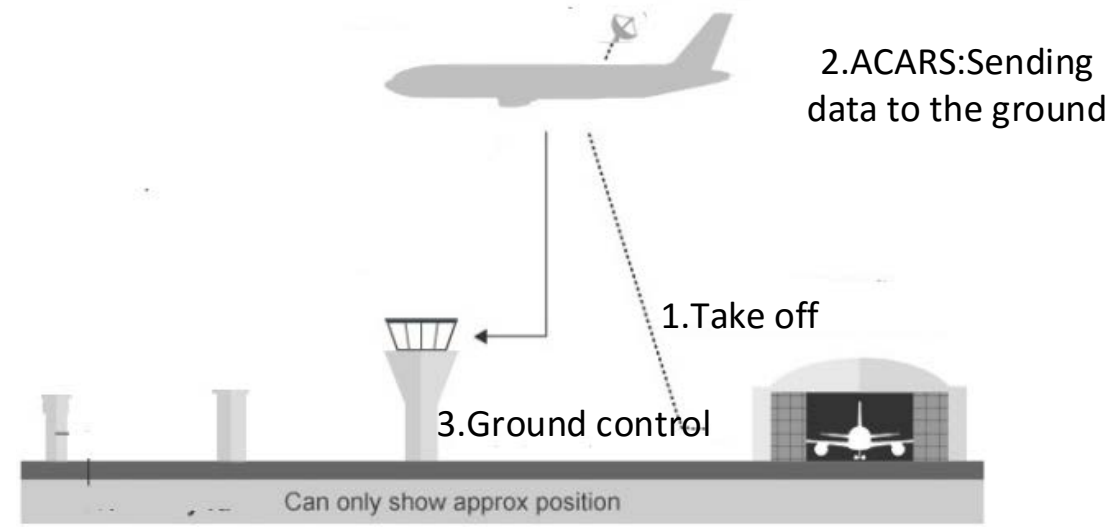

Fig.1. ACARS for communication with ground control 
There are more than 110 types of different messages which transmitted by ACARS, and these include fuel consumption, engine performance data, and aircraft position as well as free text data. All these messages are formatted in the following structure [6-7]. Therefore, ACARS is the safest but also the only reliable data link to location the aircraft real-time position in present civil aircraft technology system[ 8]. However, ACARS has the following imperfections:(1) low frequency, no timeliness: ACARS is sent every half an hour or longer, often missing. (2) monitoring passivity: the position data is sent by aircraft carried device and accept by the control personnel passively. The ground will not be able to receive a signal if the aircraft is shut down because of a mechanical failure or human individual operation.(3) No supporting for early warning of off course: the flight position is like black box process to ground control personnel during the aircraft flying process.

\section{ADS-B technology.}

With the rapid development the civil aviation industry, the resources of civilian air space facing a serious shortage and the air traffic congestion is increasing yet. Then, the traditional technology of radar surveillance and control unable to cope with today's aviation needs gradually[9]. The system of collision avoidance based on the technology of ADS-B (Automatic Dependent Surveillance-Broadcast) is sharing a wealth of aviation information from the satellite technology and the applications of digital communication, so it can reduce the difficulty of the work for pilots and the intensity for the ground commander. Since Malaysia Airlines incident, the U.S. government has asked all aircraft must be equipped with the ADS-B system in January 2020. Equipped with ADS-B aircraft can broadcast its own precise location and other data through the data chain[10]. ADS-B has high frequency, almost seconds obtain an updated location information, but it is low accuracy, and due to GPS positioning, the signal will be disturbed.

There is an efficient way to fuse ACARS and ADS-B for real-time monitoring of civil aircraft. However, it is still a challenge of aviation safety to analysis of human abnormal flight behavior during the flight. As known to the author's knowledge, there is no yet technical research to solve it. Therefore, this paper proposed the real time monitoring and early warning of pilot abnormal behavior by analyzing the real-time flight date. The next section shows details of the approach.

\section{Fusion of the Multi-Source data Information approach}

The airline's database often real-time stores flight data at every moment, such as ADS-B, ACARS, planned route data, historical route data, etc. Most of these data is only for the backup storage and will not be visible to provide real-time monitoring of the seats of the seats. Based on these data, this paper establishes a fusion data model, and designs the flight tracking alarm three core modules. As shown in the Fig.2., by fully fusing flight dynamic data, we can monitor the behavior of the aircraft in the way of real time, intelligent identification and early alarm. On the one hand, to help ground control personnel monitor the status of the aircraft. On the other hand, intelligent judgment abnormal behavior of the flight, and in a few seconds automatically give the alarm. Then the ground control personnel should contact with pilots get in touch to identify and remove abnormal. By this way, MH370 and Air France flight were impossible to missing notice about abnormal behaviors after losing contact even nearly forty minutes.

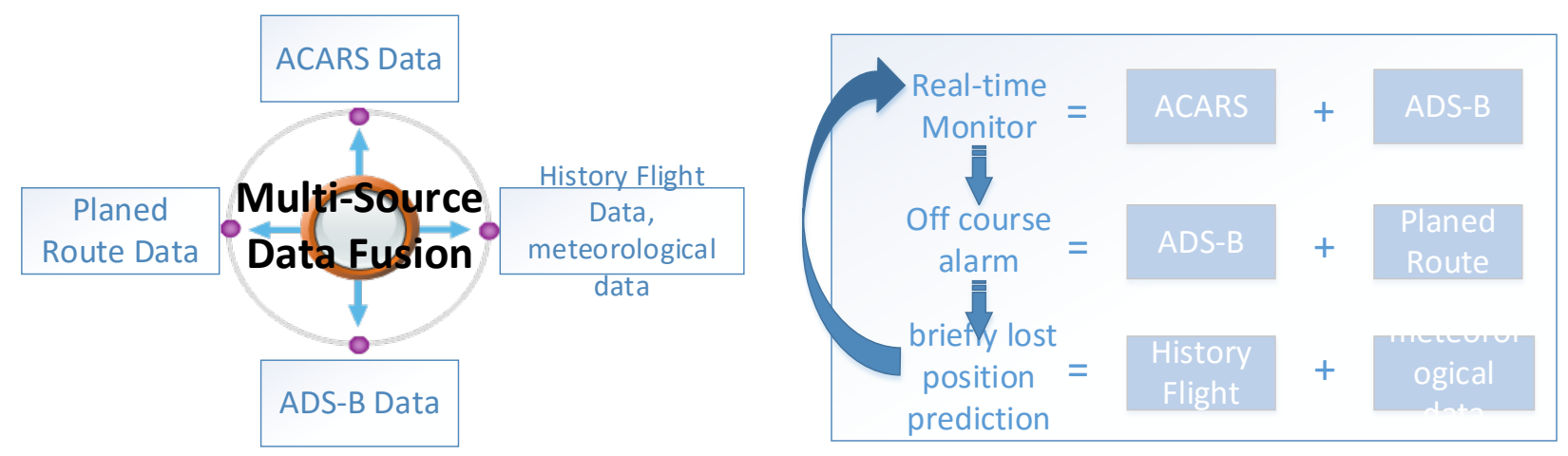

Fig.2. Multi-source data fusion 
In normal flight real-time monitoring, if aircraft flight trajectory deviates from the planned route, the alarm will help ground service personnel to contact the crew to know the details. If there are weather, signal, the fault of the equipment and other factors caused by unconventional aircraft position briefly lost contact, the multi-source data fusion model based on historical data is developed to predict the probability of location. Ground personnel do "aware" of each aircraft position and flight state at all times.

\section{(1)Visual real time monitoring model for aircraft flight path}

Under normal circumstances, the ACARS low frequency data and ADS-B data fusion visualization display, for the seat control personnel to observe the dynamics of aircraft tracks in real time. Because ACARS data has very high accuracy, so in the case of ACARS data, the use of ACARS location data, followed by ADS-B data.

\section{(2)TLC algorithm based on the model of route departure warning}

In normal, the aircraft's real-time route must be strictly follow the planned route flight. Multi source information fusion method is an intelligent early warning model for aircraft real time route and plan route. As shown in the Fig.4., flight if deviate from the planned route distance more than $\mathrm{L}$ and continued for length of a certain threshold, ground control will get alarm, then notify and contact unit personnel confirms the yaw, and continue to monitor the aircraft.

Fig.3. shows the formula, $L_{P}$ means aircraft lateral position, that is, the lateral distance of aircraft longitudinal axis and plan route line, $\mathrm{V}_{\text {y }}$ says aircraft instantaneous lateral velocity, by calculating the current position of the aircraft relative to the planning route of aircraft movement distance calculation to obtain the time.

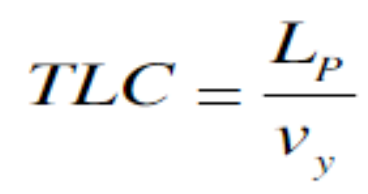

Fig.3. TLC formula

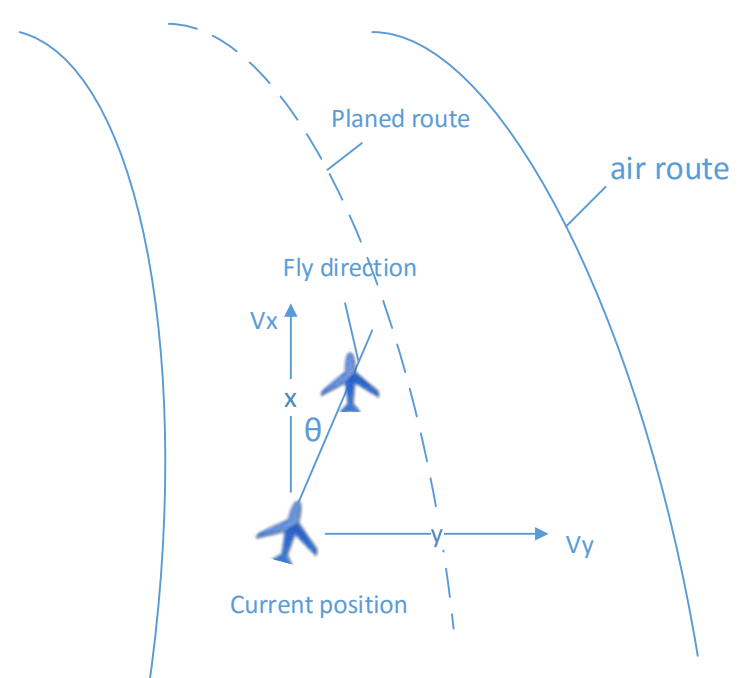

Fig.4. Departure route mode

(3) Short time lost contact track position dynamic prediction probability model

When the aircraft is in yaw, and the position data is lost due to the weather phenomenon or the signal difference, the dynamic prediction model of the short time lost track position is triggered. The model combines historical flight data, weather pilot flight record data, weather data, and gives the position of the aircraft may be predicted, such as the map. Probability model:

$\mathrm{P}=\mathrm{f}(\mathrm{D}(\mathrm{xn}), \mathrm{W}$ (yn), Pe (Zn), T (n))

Where: $\mathrm{D}(\mathrm{xn})$ : the historical route data set,

$\mathrm{W}$ (yn): regional meteorological data set

Pe (Zn): Flying habits data

$\mathrm{T}(\mathrm{n})$ : other factors

Output probability model of aircraft flight direction and position: Green is more than 60\%; 
Yellow is less than $60 \%$ and greater than $20 \%$; Red is less than $20 \%$.
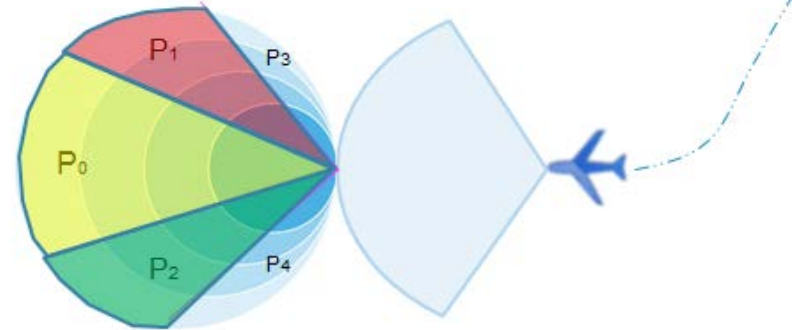

Fig.5. Analysis of short time lost contact aircraft trajectory prediction

\section{Implement and application of real-time tracking and warning system}

According to the interface of the data service platform of China Southern Airlines, we develop and implement the flight tracking alarm system. The function examples is shown as the following system screenshots.

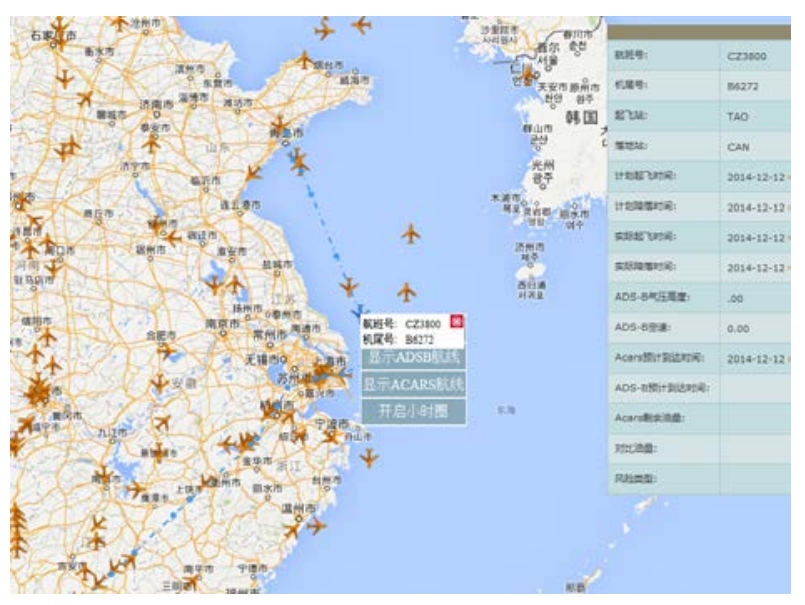

Fig.6. Real time monitoring function

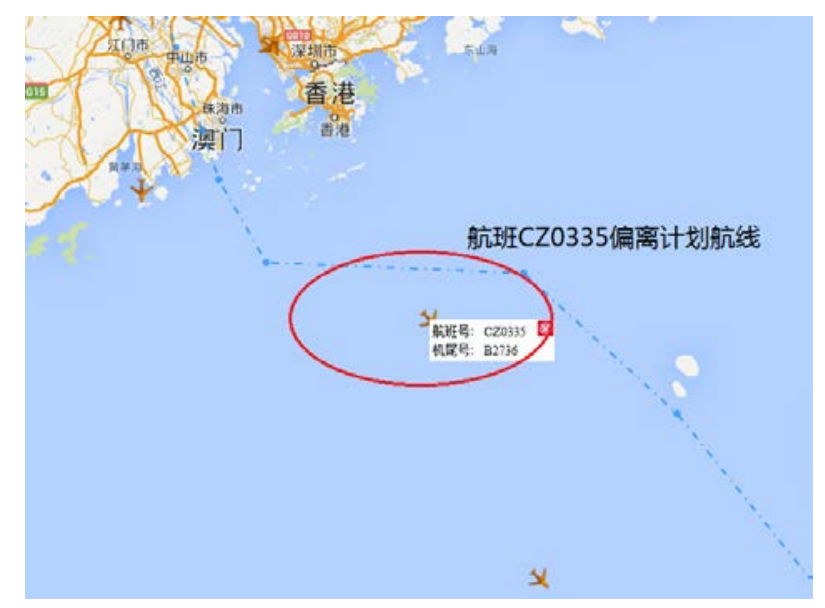

Fig.7. Off course alarm

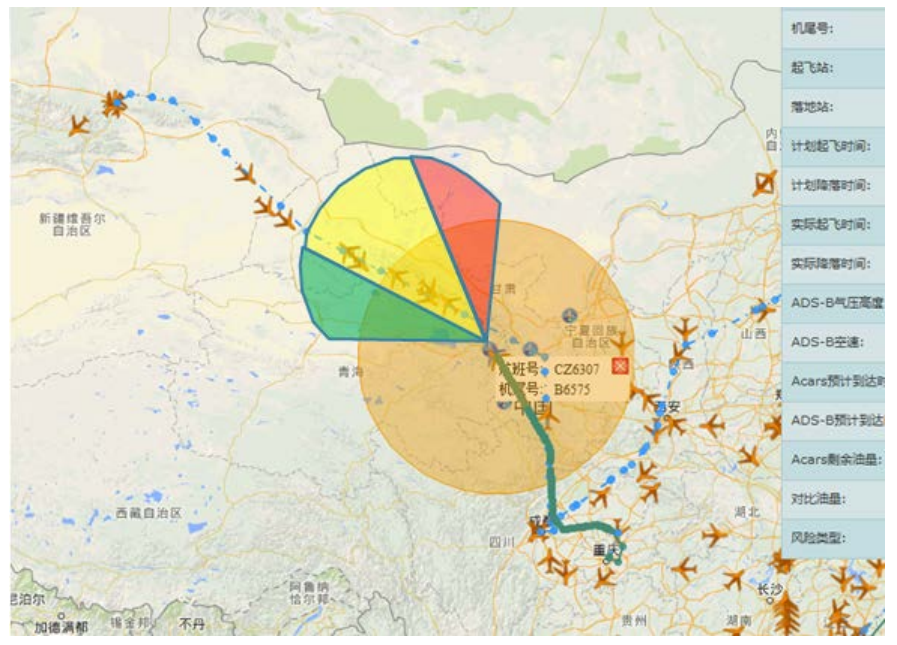

Fig.8. Transient lost contact position prediction

\section{Conclusion}

Malaysia Airlines 370 event made civil aviation aircraft safety issues become the global focus of attention, and real-time tracking and monitoring of flight trajectory has become an urgent issue to be solved. However the existing civil aircraft tracking technology lacks solution for real-time 
tracking and monitoring of the aircraft off course anomaly. To solve the issue, this paper proposed a real time tracking and monitoring approach based on fusion of multi-source data information for civil aircraft, which consists of three modules: (1) visual real-time tracking and monitoring (2) route deviation alarm (3) position prediction when lose contact in short time. Based on this approach, the flight tracking and warning system is implemented. The practical application proves that the approach and the model is effective. The main contribution of the paper is that instant found aircraft flight abnormal behavior and pre-warning intervention, maximum eliminate airliner lost contact, so that we have a chance to prevent the dangers of human errors in panic similar to Air France 447 and possibly for Malaysian airline mh370, rather than analyze what happened after a crash, and only if the black box was found. The system has been applied to China Southern Airlines to monitor air flights every day more than two thousand.

\section{Acknowledgement}

This research was sponsored by the support from Research Fund for Doctoral Program of High Education of China (No.20124307110017) and Research Project of State Key Laboratory of High Performance Computing of National University of Defense Technology (No. 201303-05).

\section{References}

[1] Jerry.J Huang, Q.S Gu, L.S ,Huang, A real-time black box system for airlines and passengers: Democratizing air safety Institute of Navigation International Technical Meeting 2015, ITM 2015, p 251-255, 2015

[2] P. Tang, S.G Zhang, L. Jin, A novel emergency flight path planning strategy for civil airplanes in total loss of thrust, Proceedings of 2nd International Symposium on Aircraft Airworthiness, p226-235, 2011.

[3] Wahid Mastura, Bouadi Hakim, Revoredo Teo, and Mora-Camino, A framework for flight guidance along air streams,2014 IEEE Chinese Guidance, Navigation and Control Conference, CGNCC 2014, p 2198-2203, January 12, 2015.

[4] Withers M R, Malina R, Gilmore C K, et al. Economic and environmental assessment of liquefied natural gas as a supplemental aircraft fuel[C]// SF2A-2013: Proceedings of the Annual meeting of the French Society of Astronomy and Astrophysics. SF2A-2013: Proceedings of the Annual meeting of the French Society of Astronomy and Astrophysics, 2013:233-236.

[5] ARINC618-5, air/ground character-oriented protocol specification, Aeronautical Radio, Inc., August 31, 2000.

[6] J.Zhang, Q.S.Zhou, X. Fang and Y.B. Zhu, Evaluation of link operation modes on air-ground VHF data link, Acta Aeronautica et Astronautica Sinica, Vol21. No.3, May 2000, 210-213.

[7] S.G Sun, ACARS data identification and application in aircraft maintenance, 1st International Workshop on Database Technology and Applications, DBTA 2009, p 255-258, 2009

[8] L.Sheng, L.Z Song, The research on ACARS digital signal demodulation method,2012 2nd International Conference on Electric and Electronics, EEIC 2012, April 21, 2012 - April 22, 2012

[9] Liu J J, Tian L Y, Wu Q X, et al. Civil Aircraft Radar Track Simulation Algorithm Based on 4D Trajectory[J]. Advanced Materials Research, 2014, 905:514-518.

[10] Estefan M. Ortiz, Ashish Babbar, Vassilis L. Syrmos,Gregory J. Clark, John L. Vian, Michael M. Arita. MultiSource Data Integration for Aircraft Health Management.IEEE Aerospace Conference, part 8, 2008 